\title{
Clinical Study \\ Presumed Cases of Mumps in Pregnancy: Clinical and Infection Control Implications
}

\author{
Svjetlana Lozo, ${ }^{1}$ Ahmed Ahmed, ${ }^{1}$ Edward Chapnick, ${ }^{2}$ Mary O'Keefe, ${ }^{3}$ and Howard Minkoff ${ }^{1,4}$ \\ ${ }^{1}$ Department of Obstetrics and Gynecology, Maimonides Medical Center, 967 48th Street, Brooklyn, NY 11219, USA \\ ${ }^{2}$ Department of Internal Medicine, Maimonides Medical Center, Brooklyn, NY 11219, USA \\ ${ }^{3}$ Department of Infection Control, Maimonides Medical Center, Brooklyn, NY, USA \\ ${ }^{4}$ Department of Obstetrics and Gynecology, Maimonides Medical Center, SUNY Downstate Medical Center, Brooklyn, \\ NY 11203, USA \\ Correspondence should be addressed to Howard Minkoff, hminkoff@maimoniesmed.org
}

Received 2 November 2011; Accepted 21 November 2011

Academic Editor: Lu-Yu Hwang

Copyright (C) 2012 Svjetlana Lozo et al. This is an open access article distributed under the Creative Commons Attribution License, which permits unrestricted use, distribution, and reproduction in any medium, provided the original work is properly cited.

\begin{abstract}
Recently, a mumps outbreak in New York and New Jersey was reported by the Centers for Disease Control and Prevention (CDC). Subsequently, the dissemination of the disease was rapid, and, from June 28th 2009 through January 29th 2010, a total of 1,521 cases of mumps were reported in New York and New Jersey. Seven presumed cases occurred in pregnant women cared for at our institution. Mumps diagnosis as per the NYC Department of Health and Mental Hygiene was based on clinical manifestations, particularly parotitis. Prior immunizations with mumps vaccine and negative IgM were not adequate to rule out mumps infections. All of our seven patients had exposure to mumps in either their household or their community, and some of the them had symptoms of mumps. Due to the difficulties in interpreting serologies of these patients, their cases led to a presumed diagnosis of mumps. The diagnosis of mumps lead to the isolation of patients and health care personnel that were in contact with them. In this paper, we detail the presenting findings, diagnostic dilemmas and infection control challenges associated with presumed cases of mumps in pregnancy.
\end{abstract}

\section{Introduction}

Mumps is an acute infectious disease, caused by an RNA paramyxovirus. Humans are the only natural hosts, and the virus is spread by contact with infected respiratory droplets. Patients with mumps are usually contagious for two days prior to gland swelling and up to five days after the swelling appears. The course of mumps tends to begin with nonspecific symptoms such as myalgia, headaches, anorexia, malaise, and high fever. Unilateral or bilateral parotid gland enlargement tends to present 24 hours after initial symptoms. Typical acute parotitis occurs in only about $30-40 \%$ of cases, while $15-20 \%$ of infections are completely asymptomatic, and up to $50 \%$ of infections are associated with nonspecific or primary respiratory symptoms. Inapparent infection or completely asymptomatic mumps infection more commonly occur in adults than in children [1].

Although disease is generally mild and self-limited, occasional complications may ensue; ten percent of patients develop aseptic meningitis, and less common complications include encephalitis, permanent deafness, orchitis, and pancreatitis [2]. Vaccination has been key to the prevention of those complications, and, as of 1998, 82 countries had established childhood immunization programs [3]. Over the last several decades, there have been few reports of mumps epidemics in the United States (US).

Recently, a mumps outbreak in New York and New Jersey was reported by the Centers for Disease Control and Prevention (CDC) [4]. The index patient was an 11-year-old boy who traveled to the United Kingdom, where over seven thousand cases of mumps have been reported since 2004 [5]. After his return to the US, the patient traveled to a summer camp for observant Jewish boys in upstate New York. Subsequently, the dissemination of the disease was rapid, and from June 28th 2009 through January 29th 2010, a total of 1,521 cases of mumps were reported in New York and New Jersey [4]. Seven presumed cases occurred in pregnant women cared for at our institution. In this paper, we report this series of women, 
discuss lessons learned from their care, and review the recent literature. We highlight the difficulty in making definitive diagnoses, the limitations of serology and vaccination, and the potential adverse impact on staffing consequent to exposure to presumed cases.

\section{Cases}

Between August 2009 and early January 2010, 40 cases of confirmed or suspected mumps were seen at Maimonides Medical Center, seven of whom were women in the antepartum, intrapartum, or postpartum period. Per the recommendations of the New York City Department of Health and Mental Hygiene (NYC DOHMH), the case definition of mumps included anyone who presented with the acute onset of unilateral or bilateral tender, self-limited, swelling of parotid, or other salivary glands lasting two or more days without other apparent cause [6]. Additionally, all women presenting to the obstetrical triage area during the outbreak were asked about recent exposure to mumps. This strategy was based in large part on the failure of serostatus to accurately reflect immune status, and the known failure rates of vaccine (vide infra). Thus, in a community in the midst of an epidemic, any patient who lived in the borough of Brooklyn, and who had neck swelling without other apparent cause, was considered to have mumps [7].

The first case was a 31-year-old multiparous woman who presented in labor at 35-weeks gestation with spontaneous rupture of membranes. During the initial examination, the patient reported that she had been diagnosed with mumps a few weeks earlier after one of her sons presented with symptoms. Since we could not confirm her diagnosis, and we did not know the date of her last exposure, a decision was made to place the patient in a private room with droplet precautions, and she was induced with cervidil. After delivery, the patient was continued on droplet precautions. No confirmatory testing was performed since it would not have affected management. The second patient, a 45-year-old para 13, presented to the hospital at 39-weeks gestation for a repeat cesarean section. On postpartum day three, she complained of parotid gland swelling. As per the patient, she did not have any known contact with any individuals with mumps. The patient was afebrile but had parotid gland tenderness. The patient was placed on droplet precautions. Subsequent serum mumps IgM antibody was negative and IgG was 2.37 (immune).

The third patient, a 38 year old para 12 at 41 weeks and one-day gestation, was seen in labor and delivery in active labor and reported that she had been diagnosed with a mumps infection by her PMD three days prior, based upon her clinical presentation. The patient was placed on droplet precautions, given a face mask to be used when in contact with her infant, and was discharged home two days postpartum. In this case, mumps IgG and IgM levels were not sent because she had previously been diagnosed, and her management would not have been affected. The fourth patient, a 37 year old para 5 at term, presented to labor and delivery in active labor. Two days postpartum, the patient complained of swelling of her jaw and tenderness. She reported that a diagnosis of mumps had been suspected in her 10-year-old son a few days earlier. Blood drawn at the time of symptom onset subsequently returned as IgG 2.27 and IgM negative. During the investigation period, the patient was placed on droplet precautions and monitored closely. The fifth patient, a 29 year old para six at 37-weeks and 5-days gestation, presented to labor and delivery with signs of preeclampsia. The patient was given magnesium and induced with cervidil. The patient reported that three of her children at home were diagnosed with mumps. She complained of weakness and had parotid gland tenderness and swelling, and a mild fever. The patient was placed on droplet precautions. The IgG antibody was 2.43 , and IgM was negative. She delivered 24 hours after her admission and was discharged home a few days later.

Two additional pregnant patients with suspected mumps infection were seen in the emergency room (ER), one at 32 weeks of gestation and the other at 10 weeks of gestation. The first was a 24 year old para 1 in the third trimester, who presented with URI symptoms, difficulty swallowing, and parotid gland tenderness. She was discharged home with precautions. Mumps IgG antibody was 1.13 , and IgM was negative. The second patient was a 22 year old para 1 in the first trimester who presented with nausea and vomiting. The patient reported that she had been diagnosed with mumps by her physician one week previously. While in the ER, she was placed on droplet precautions, given IV hydration and ondansetron, and was discharged home a few hours later.

In all cases, providers who had been in contact with the source patients before the patients were placed on droplet precautions were queried about their immunization status. If they did not have evidence of two prior MMRs, a physician documented history of mumps or serologic evidence of immunity, mumps IgG testing was performed. While the results were pending, they were furloughed. If the serology results were negative, they were removed from the workplace for the entire incubation period, from days 12 through 25 after exposure. For a brief period during the outbreak, the NYC DOHMH did not consider serologic evidence of IgG to be a marker of immunity and recommended that exposed employees unable to produce proof of receipt of 2 MMR vaccines or a physician documented history of mumps be furloughed for the incubation period.

\section{Discussion}

During the outbreak period, the NYC DOHMH recommended that all patients be screened for symptoms of mumps as well as for a history of recent exposure at the time of admission. This was essential for preventing transmission of mumps to patients and staff within the hospital. We report a series of obstetrical patients who were suspected of having mumps or who had recent exposure during that epidemic. For reasons discussed below, all cases were diagnosed based on clinical presentation, independent of IgG or IgM levels. Postpartum mothers who were suspected of having mumps were advised to wear a mask within 3-6 feet of their infants.

Part of the difficulty in managing these cases was the realization that assessment of vaccination status and serostatus 
is an inexact predictor of an individual's mumps immune status. Current mumps immunization recommendations are for a first dose of Measles Mumps Rubella (MMR) vaccine at age 12-15 months and a second dose between the ages of four and six. A child is considered to have adequate immunity when there has been documented administration of two doses of live virus vaccine or laboratory evidence of immunity to disease. The effectiveness of mumps vaccine has been estimated to be $73-91 \%$ after the first dose of vaccine, and 76-95\% after two doses of vaccine. [8] However, recent mumps outbreaks have been seen in fully immunized populations, [9] pointing out the potential failure of vaccines to confer protection from disease. Data also suggest that there are limitations to the use of serologic tests for diagnosing mumps, raising the question of how to interpret those tests in the setting of a clinical presentation compatible with mumps [4].

Pregnant women with mumps face concerns beyond those of the general population. Ornoy reported that mumps infection in pregnancy increased the risk of embryonic and fetal death as well as spontaneous abortion, but did not seem to have any relation to fetal congenital anomalies [3]. However, the patient population that was reviewed was extremely small. In another study of five women volunteers who were scheduled to have second trimester therapeutic abortions, and who were immunized by the Jeryl-Lynn strain of attenuated mumps virus $7-10$ days before their scheduled abortion, mumps virus was detected in $2 / 5$ placentas but in none of the fetuses [10]. However, it is possible that the interval between immunization and abortion was too short to allow for viral replication in fetal organs. Jones et al. addressed the issue of mumps infection in the perinatal period in 1980 [11]. In that case series, three women with symptomatic mumps during labor, with three different fetal outcomes, were reported. One infant had swelling in the parotid area and a positive mumps skin test at 42 days of age. A second infant developed pneumonia on day 7 and had to be hospitalized and intubated. That infant subsequently presented with upper respiratory tract complications in the first year of life. The third infant had no evidence of mumps and never developed symptoms. In all three of these cases, infants reached normal developmental milestones and had no further complications.

More serious complications of mumps infection include aseptic meningitis (occurring asymptomatically in 50-60\% of cases), symptomatic meningitis (up to $15 \%$ ), encephalitis (less than 2 per 100,000), myocarditis (3-15\%), pancreatitis $(2-5 \%)$, deafness $(1$ in 20,000$)$, and inflammation of ovaries (up to 5\%), arthritis, and nephritis, which are less common, and on average one death per year from mumps from 19801999 [12]. Desai described a primigravida at term with rupture of membranes and acute renal failure with a clinical diagnosis of mumps. The patient was managed by immediate delivery by cesarean section and given IV steroids as well as diuretics for the management of suspected mumps nephritis. The patient recovered but the neonate expired soon after delivery, and no investigation was performed to determine if the neonate had mumps [13].

Despite these risks, pregnant women are frequently unvaccinated. Haas et al. in 2005 found that current screening and vaccine programs left pregnant women susceptible to measles, mumps, and rubella and concluded that there is a need for a more comprehensive viral screening program to ensure immunity and adequate vaccination, and to establish better care in the prenatal period. In their study, $16.3 \%$ of women were susceptible to mumps infection, and one third of women in their study were found to lack immunity to rubella, rubeola or mumps [14]. Coonrod et al. have suggested that immunizations be a part of preconception care [15]. Hepatitis B and MMR vaccine are already recommended as part of preconception care, because of the benefit of giving these immunizations before pregnancy, and their effectives in preventing maternal disease and vertical transmission [15].

Mumps infection can be diagnosed by isolation of mumps virus or nucleic acid from clinical specimens, by observing a rise in mumps-specific immunoglobulin titers between acute and convalescent serum samples or by a positive serologic test for mumps IgM in serum or saliva [16]. Mumps-specific IgM can be detected on the 11th day after exposure and is usually present by the time clinical illness develops. IgG levels offer evidence of prior exposure but do not correlate well with immunity [17]. Currently, the gold standard for diagnosis of mumps is viral culture [9]. However, that test is not readily available for clinical use.

In addition to the difficulty in making the diagnosis of mumps in the clinical setting, another issue raised by our experience was the need to address the infectious nature of the illnesses and the susceptibility of other patients and staff to potentially infectious source patients. CDC guidelines advise placing patients with suspected infection on droplet precaution. What is less clear is how to manage patients with symptoms compatible with mumps infection who have had two MMR vaccines in the past, and who have IgG antibody. As noted above, the vaccine is imperfect $(76-95 \%$ effective), and circulating IgG levels are not reliable markers of adequate immunity [18]. IgM is also unreliable, since there might only be a transient peak in IgM in the presence of IgG such that the absence of IgM does not preclude acute infection. Because of these uncertainties, the putative mumps patients had a disruptive impact on staffing. Healthcare workers with a history of vaccination or evidence of $\operatorname{IgG}$ antibody to mumps were regarded as immune. However, some individuals can be $\operatorname{IgG}$ negative even after vaccination, and since evidence of mumps vaccination status is not a condition of employment, many physicians and nurses were unaware of their status. Among staff without serologic evidence of immunity, evidence of two MMR vaccines or a physician note confirming prior mumps infection were accepted as evidence of immunity. Healthcare workers without such documentation were removed from the workplace for the entire incubation period, 12-25 days after exposure. During a brief period, the NYC DOHMH did not consider a positive IgG as evidence of immunity, and these employees were also removed from the workplace.

When mumps becomes endemic in a community, pregnant patients being seen in a hospital or clinic should be routinely asked about mumps exposure and symptoms. If infection is suspected, the patient should immediately be placed 
in isolation and put on droplet precautions. It may also be worth incorporating screening for mumps immunity at the time of employment offering nonimmune individuals additional booster vaccines.

Finally, in a mumps advisory disseminated a few months after the epidemic, the NYC DOHMH suggested that the diagnosis of mumps should be based on clinical manifestations, particularly parotitis. It was also mentioned that patients who had been immunized with mumps vaccine can still be infected with mumps, and the diagnosis should not be ruled out on the basis of negative IgM. The recommendations that the NYC DOHMH made, and which are now followed at our institution, and which we believe would be useful on any obstetrical service facing a similar circumstance are the following:

(1) Patients who present with parotitis, jaw pain, fever, or orchitis should be isolated for five days, and mumps serology should be sent unless another cause of parotitis is determined

(2) Patients who might have been in close contact with mumps virus should be excluded from work or school for 25 days after exposure or until they are vaccinated

(3) Health care workers should be given appropriate attire (mask) when working with suspected mumps patients. Exposed health care workers who have never been vaccinated or who do not have evidence of mumps immunity should stay at home from days $12-$ 25 after exposure, even if they receive a first dose of vaccine after exposure. Workers who have received one dose of vaccine in the past could continue working, but should get a second dose of the MMR vaccine 28 days after the first one. Health care workers with serologic evidence of immunity can continue their daily work duties despite their exposure to virus [7].

These recommendations call for routinely using diagnostic tests in nonepidemic situations. However, in an epidemic situation, such as the one we describe, it became apparent that tests had limitations, including the turnaround time needed for obtaining results. Thus, decisions about hospital infection control may often have to be made before results become available.

\section{References}

[1] A. Ornoy and A. Tenenbaum, "Pregnancy outcome following infections by coxsackie, echo, measles, mumps, hepatitis, polio and encephalitis viruses," Reproductive Toxicology, vol. 21, no. 4, pp. 446-457, 2006.

[2] A. M. Galazka, S. E. Robertson, and A. Kraigher, "Mumps and mumps vaccine: a global review," Bulletin of the World Health Organization, vol. 77, no. 1, pp. 3-14, 1999.

[3] "CDC Update: Mumps Outbreak- New York and New Jersey, June 2009-January 2010," Morbidity and Mortality Weekly Report, vol. 59, no. 5, pp. 125-129, 2010.

[4] Center for Disease Control and Prevention (CDC 2011), "Facts About Mumps," http://www.cdc.gov/mumps/.
[5] J. F. Jones, C. G. Ray, and V. A. Fulginiti, "Perinatal mumps infection," Journal of Pediatrics, vol. 96, no. 5, pp. 912-914, 1980.

[6] D. Haas, C. Flowers, and C. Cogndon, "Rubella, rubeola and mumps in pregnant women susceptibilities and strategies for testing and vaccinating," American College of Obstetrics and Gynecology, vol. 106, no. 2, pp. 295-300, 2005.

[7] R. K. Gupta, J. Best, and E. MacMahon, "Mumps and the UK epidemic 2005," Clinical Review, BMJ, vol. 330, pp. 1132-1135, 2005.

[8] P. Mead, W. D. Hager, and S. Faros, Protocols for Infectious Disease in Obstetrics and Gynecology, Blacwell Science Inc, 2000.

[9] T. Yamauci, C. Wilcson, and J. W. Geme, "Transmission of live, attenuated virus to the human placenta," The New England Journal of Medicine, vol. 290, pp. 710-712, 1974.

[10] P. Dasari, "Maternal and perinata outcome in mumps complicating pregnancy," The Internet Journal of Gynecology and Obstetrics, vol. 13, no. 2, 2010.

[11] National Foundation for Infectious Diseases, "Facts About Mumps for Adults," 2009, http://www.nifd.net/.

[12] A. Hviid, S. Rubin, and K. Muhlemann, "Mumps," The Lancet, vol. 371, pp. 932-944, 2008.

[13] V. Kancherla and C. Hanson, "Mumps resurgence in the United States," Journal of Allergy and Clinical Immunology, vol. 118, no. 4, pp. 938-941, 2006.

[14] G. Monif, "Maternal mumps infection during gestation: observations on the progeny," American Journal of Obstetrics and Gynecology, vol. 119, no. 4, pp. 549-551, 1974.

[15] State of New York Department of Health, "Mumps Advisory," 2010.

[16] “Mumps epidemic United Kingdom, 2004-2005,” MMWR Morbidity Mortality Weekly Report, vol. 55, no. 7, pp. 173-175, 2006.

[17] D. Coonrod, B. W. Jack, K. A. Boggess et al., "The clinical content of preconception care: immunizations as part of preconception care," American Journal of Obstetrics and Gynecology, vol. 199, no. 6, pp. S290-S295, 2008.

[18] S. A. Plotkin and S. A. Rubin, "Mumps vaccine," in Vaccines, S. A. Plotkin and W. A. Orenstein, Eds., pp. 435-465, Saunders, Philadelphia, Pa, USA, 5th edition, 2008. 


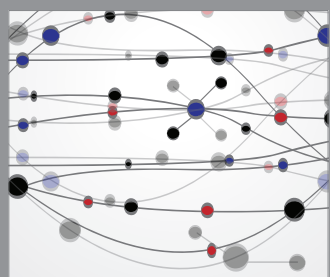

The Scientific World Journal
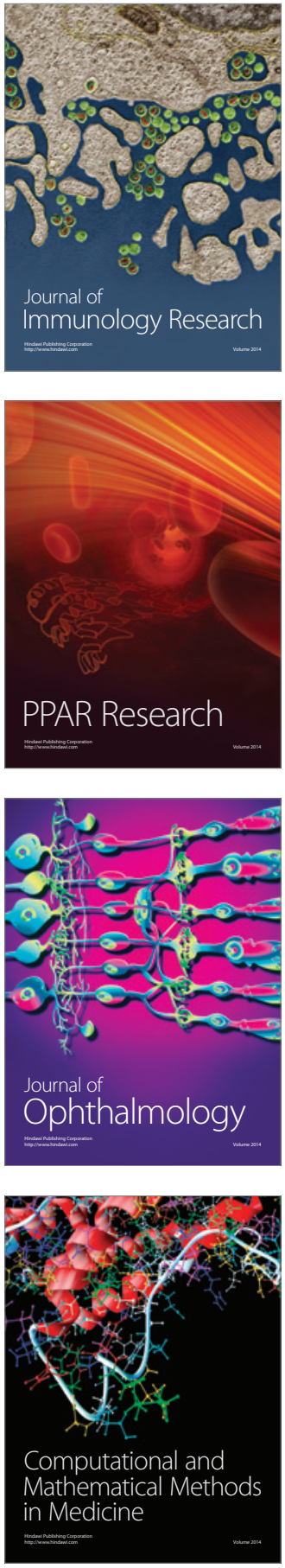

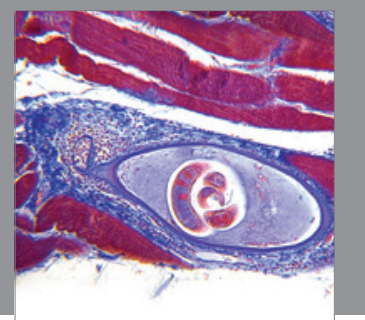

Gastroenterology

Research and Practice
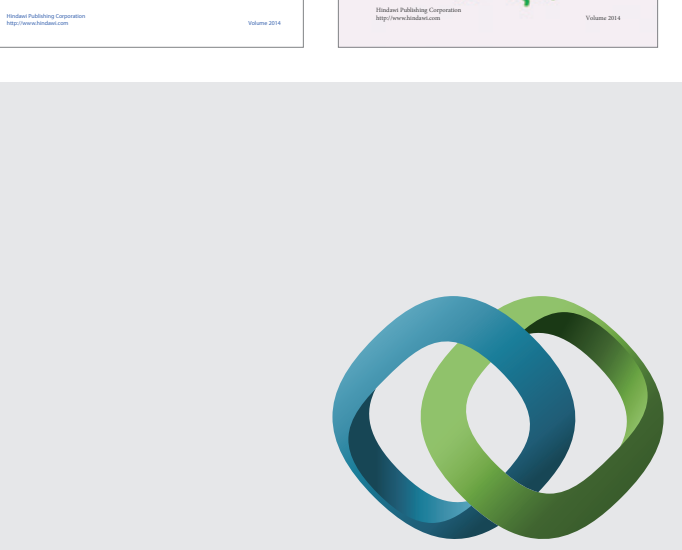

\section{Hindawi}

Submit your manuscripts at

http://www.hindawi.com
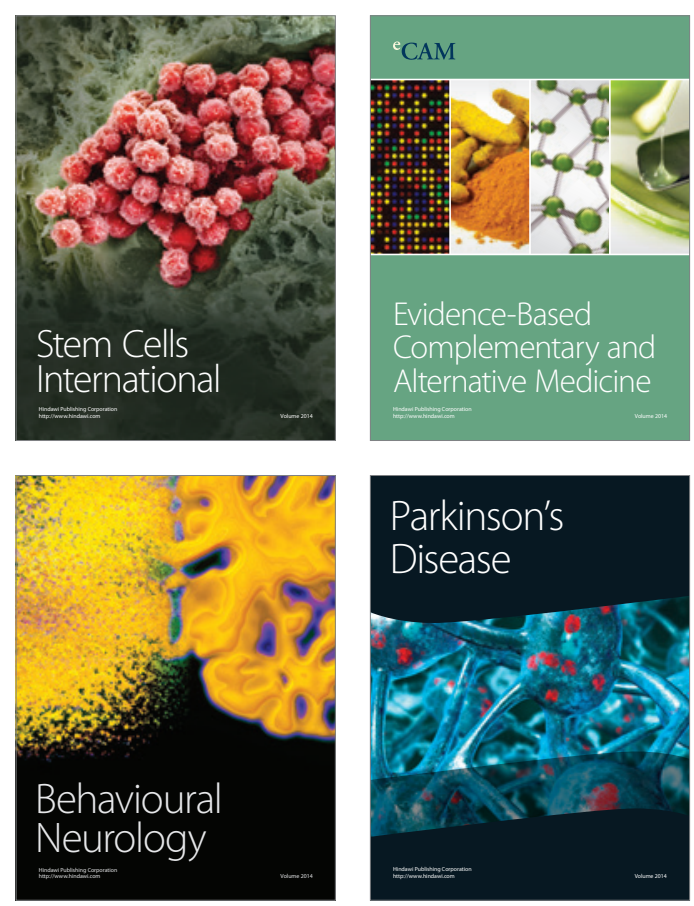

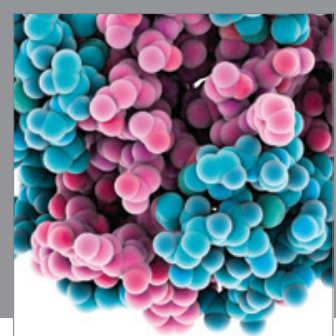

Journal of
Diabetes Research

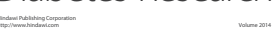

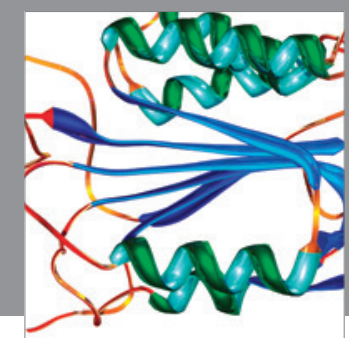

Disease Markers
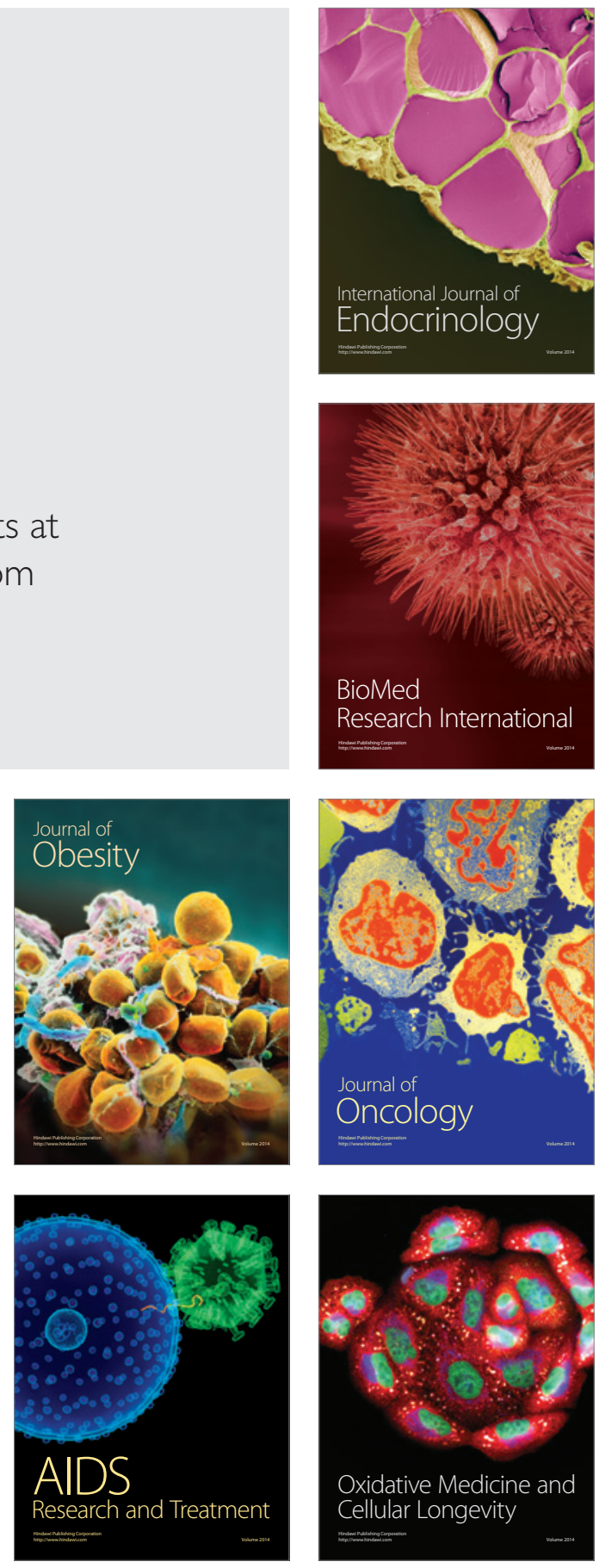